\title{
Gender Difference Analysis of Chinese Han Youth Based on Fingerprint Ridge Density
}

\author{
Huawei Xie ${ }^{1, *}$, Zifeng Lin $^{2}$ \\ ${ }^{1}$ Department of Forensic Science, Fujian Police College, Fuzhou, PR China 350007 \\ ${ }^{2}$ Haikou public security bureau, Haikou, PR China 570100 \\ ${ }^{*}$ Corresponding author at: Department of Forensic Science, Fujian Police College, Shoushan Road 59, Cangshan District, Fuzhou 350007, \\ PR China.
}

\begin{abstract}
Objective To discuss whether there are differences in fingerprint ridge density between the males and the females and what the specific differences are. Method A total of 120 males and 120 females (all were college students in Fujian) were selected, and the fingerprint ridge densities of these 240 Han youths were counted. According to Acree method, the radial upper part of the core region of the fingerprint center was selected for analysis, because the fingerprint pattern types in this region all presented similar ridges. This method was used to isolate the ridge in a certain area, so as to facilitate the counting. A $5 \mathrm{~mm} \times 5 \mathrm{~mm}$ grid was designated as a counting area on the middle radial part of the fingerprint for identification. Fingerprint ridges in the unit area were counted, then the average was calculated, and finally SPSS software was used to process and analyze all the data obtained. Result For the females, the average ten-fingerprint ridge density was basically above 14 ridges $/ 25 \mathrm{~mm}^{2}$; and for the males, it was basically below 14.5 ridges $/ 25 \mathrm{~mm}^{2}$. Conclusion There are some differences in the density of ten-fingerprints between men and the females, and the females' is generally higher than that of the males.
\end{abstract}

\section{Introduction}

In the traditional investigation, the fingerprint identification is mainly for individual identification and criminal suspects screening. However, in the absence of the fingerprint of the criminal as the comparison object, even if a large number of valuable fingerprints are obtained at the scene, the inspection and comparison is not significant. [1] Nowadays, the scientific research conducted in criminal court is mainly aimed at discovering the most useful information at the deepest level when facing fixed biological evidence, so as to further provide more scientific and practical services for cases in criminal court. Therefore, how to find more useful information from the obtained fingerprints and provide more valuable clues for criminal investigation cases becomes a very meaningful research topic. Relevant investigation data at home and abroad show that the incidence of the most basic types of fingerprint patterns is closely related to ethnicity, race, region, gender, and blood type. Based on this theory, this study attempts to use the density of fingerprint ridge to explore the gender difference, thereby deeply digging the evidence value of the fingerprint at the scene, so as to provide directions for the detection of criminal investigation cases. Especially when fingerprints have no comparison value, if the gender of the persons left their fingerprints is concluded based on the fingerprint ridge density, combined with public security big data, such as video surveillance (male or female appearing in a specific period of time), mobile phone signals (mobile phone users are male or female), it can provide direction for investigation, reduce workload and improve case detection efficiency.

\section{Experiment}

\subsection{Preparations}

The experimental materials: palm print box, magnifying glass $(8 \mathrm{x})$, a notebook with small grids of $5 \mathrm{~mm} \times 5 \mathrm{~mm}$. A random sample of 120 male college students and 120 female college students were selected. They were all young Han population in Fujian. The study was conducted separately with explanation and verbal notification of informed consent. Subjects were asked to wash their hands before printing and 10 fingerprints were obtained by planar printing using the ink printing method. The subjects were asked to keep their arms relaxed and roll their fingers in both directions in a palm-print box to obtain a 10-finger fingerprint for the next count analysis. [2]

\subsection{Method}

Acree was used to research and analyze the fingerprints

${ }^{*}$ Corresponding author: Tel: +86 13850100062;

fax: +86059188037670. E-mail: momoxhw@163.com 
in the delineated area. [3] The radial upper part of the core region of the fingerprint center was selected for analysis, because the fingerprint pattern types in this region all presented similar ridges. [4] This method isolated the ridge line in a certain area, which was convenient for the counting. A $5 \mathrm{~mm} \times 5 \mathrm{~mm}$ area was designated as a counting area on the radial part of the middle of the fingerprint for fingerprint identification. (see Figure 1)

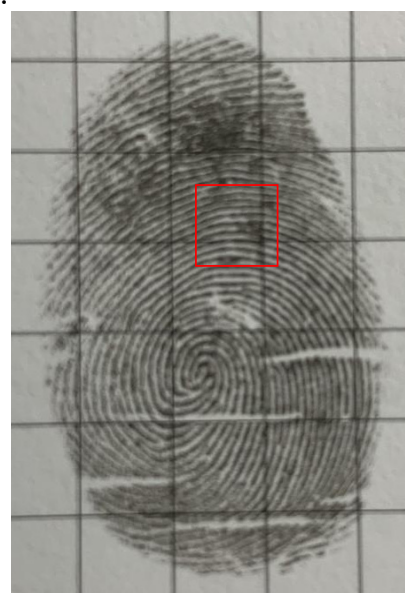

Figure 1 The radial part of the middle of the fingerprint (left thumb)

\subsection{Steps}

The collected 240 samples were placed in the $5 \mathrm{~mm} \times 5 \mathrm{~mm}$ grids to calculate the quantity of fingerprints, and the density of the fingerprint ridge inside the grid can be obtained. Among the large data obtained, the density was calculated first as follow: the right thumb, right index finger, right middle finger, right ring finger, right

Table 1 Distribution differences of ten-fingerprint fingerprint density values between different genders

\begin{tabular}{lccccc}
\hline & Thumb & Index & Middle & Ring & Pinky \\
\hline Male: & & & & & \\
Right (Max.) & 17 & 19 & 18 & 19 & 18 \\
Left (Max.) & 21 & 18 & 20 & 18 & 19 \\
Right (Min.) & 9 & 9 & 9 & 9 & 10 \\
Left (Min.) & 10 & 10 & 10 & 11 & 10 \\
Female: & & & & & \\
Right (Max.) & 18 & 18 & 21 & 21 & 18 \\
Left (Max.) & 19 & 19 & 20 & 20 & 19 \\
Right (Min.) & 11 & 11 & 11 & 12 & 11 \\
Left (Min.) & 12 & 11 & 11 & 11 & 11 \\
\hline
\end{tabular}

Table 2 Statistics of the density of ten-fingerprint ridge

\begin{tabular}{lllccc}
\hline Gender & Max. & Min. & Average & Mid-value & SD \\
& & & & & \\
\hline Male & 16.2 & 11.3 & 12.87 & 12.7 & 1.5876 \\
Female & 17.5 & 12.3 & 14.746 & 14.7 & 1.6445 \\
\hline
\end{tabular}

pinky finger, left thumb, left index finger, left middle finger, left ring finger, and left little finger; then the average of the density of each finger and the average density of ten-fingerprint were obtained by SPSS software. Based on the average density of ten-fingerprints, a table was created to show the trend of male and female fingerprints, and the results were obtained.

\subsection{Pretreatment}

The ten-fingerprints of 10 males and 10 females were separately collected and the average densities were compared. The results showed that the ten-fingerprint density of females was greater than that of males. According to statistics, the difference was statistically significant.

\subsection{Statistics}

During studying the fingerprint ridge, the specific method was as follow: use an magnifying glass (8x), count from the upper left corner of the defined area until the diagonally opposite, mark the 2 sticks, dots, and the starting points as 0 , mark the small holes, small bridges, and small hooks as 1, and mark the combination and divergence as 2; and then use SPSS software to perform statistical analysis on the data of fingerprints obtained in each sample. The likelihood ratio (LR) was calculated based on Bayes' theorem. The likelihood ratio (LR) was calculated based on Bayes' theorem, and the posterior probability inference of gender based on the ridge density was obtained. The results were shown in Table 1 , Table 2, Table 3, Table 4, Table 5, Table 6. 
Table 3 Statistics of the extreme values of the density of the ten-fingerprint ridge

\begin{tabular}{lcc}
\hline Mean fingerprint density & Male & Female \\
& & \\
$10 \leq X R<11$ & 1 & 0 \\
$11 \leq X R<12$ & 20 & 0 \\
$12 \leq X R<12.5$ & 22 & 1 \\
$12.5 \leq X R<13$ & 30 & 6 \\
$13 \leq X R<13.5$ & 18 & 9 \\
$13.5 \leq X R<14$ & 15 & 11 \\
$14 \leq X R<14.5$ & 6 & 21 \\
$14.5 \leq X R<15$ & 5 & 27 \\
$15 \leq X R<15.5$ & 1 & 17 \\
$15.5 \leq X R<16$ & 0 & 10 \\
$16 \leq X R<17$ & 2 & 13 \\
$X R \geq 17$ & 0 & 5 \\
Total & 120 & 120 \\
& &
\end{tabular}

Note: $\mathrm{XR}=$ The average density of ten- fingerprint

Table 4 XR: the average density of the ten-fingerprint ridge of the males and the females

\begin{tabular}{|c|c|c|c|c|c|}
\hline & Thumb & Index & Middle & Ring & Pinky \\
\hline \multicolumn{6}{|l|}{ Male: } \\
\hline Right & 12.5416 & 12.5286 & 12.9500 & 13.3786 & 12.4643 \\
\hline $\begin{array}{l}\text { Left } \\
\text { Female: }\end{array}$ & \multicolumn{4}{|c|}{ Female: } & 12.9071 \\
\hline Right & 14.4571 & 14.2857 & 15.2428 & 15.2928 & 14.7500 \\
\hline Left & 14.3785 & 14.6143 & 15.0428 & 15.2000 & 14.6643 \\
\hline
\end{tabular}

Table 5 XR: the average of the density of the five-fingerprint ridge the males and females

\begin{tabular}{llllll}
\hline & Thumb & Index & Middle & Ring & Pinky \\
\hline Male: & 12.62495 & 12.61844 & 13.06785 & 13.30714 & 12.6857 \\
Female: & 14.4178 & 14.45 & 15.1428 & 15.2464 & 14.70715 \\
\hline
\end{tabular}

Table 6 Posterior probability and likelihood ratio of ten- fingerprint density

\begin{tabular}{|c|c|c|c|c|c|}
\hline \multirow{2}{*}{$\mathrm{XR}$ (Average density) } & \multicolumn{2}{|c|}{ Density probability } & \multicolumn{2}{|c|}{ Likehood ratio } & \multirow{2}{*}{$\begin{array}{l}\text { Dominance } \\
\text { tendency support } \\
\text { Male Female }\end{array}$} \\
\hline & Male & Female & Male & Female & \\
\hline $10 \leq X R<11$ & 1.00 & 0.01 & 100.00 & 0.010 & $0.99>0.01$ \\
\hline $11 \leq X R<12$ & 1.00 & 0.17 & 5.882 & 0.170 & $0.85>0.15$ \\
\hline $12 \leq X R<12.5$ & 0.95 & 0.21 & 4.625 & 0.216 & $0.82>0.18$ \\
\hline $12.5 \leq X R<13$ & 0.80 & 0.34 & 2.379 & 0.420 & $0.70>0.30$ \\
\hline $13 \leq X R<13.5$ & 0.64 & 0.38 & 1.687 & 0.593 & $0.63>0.37$ \\
\hline $13.5 \leq X R<14$ & 0.57 & 0.44 & 1.283 & 0.779 & $0.56>0.44$ \\
\hline $14 \leq X R<14.5$ & 0.32 & 0.75 & 0.427 & 2.344 & $0.30<0.70$ \\
\hline $14.5 \leq X R<15$ & 0.32 & 0.81 & 0.389 & 2.569 & $0.28<0.72$ \\
\hline $15 \leq X R<15.5$ & 0.18 & 0.93 & 0.197 & 5.083 & $0.16<0.84$ \\
\hline $15.5 \leq X R<16$ & 0.08 & 1.00 & 0.080 & 12.500 & $0.07<0.93$ \\
\hline $16 \leq X R<17$ & 0.21 & 0.86 & 0.247 & 4.045 & $0.20<0.80$ \\
\hline$X R \geq 17$ & 0.04 & 1.00 & 0.040 & 25.000 & $0.04<0.96$ \\
\hline
\end{tabular}




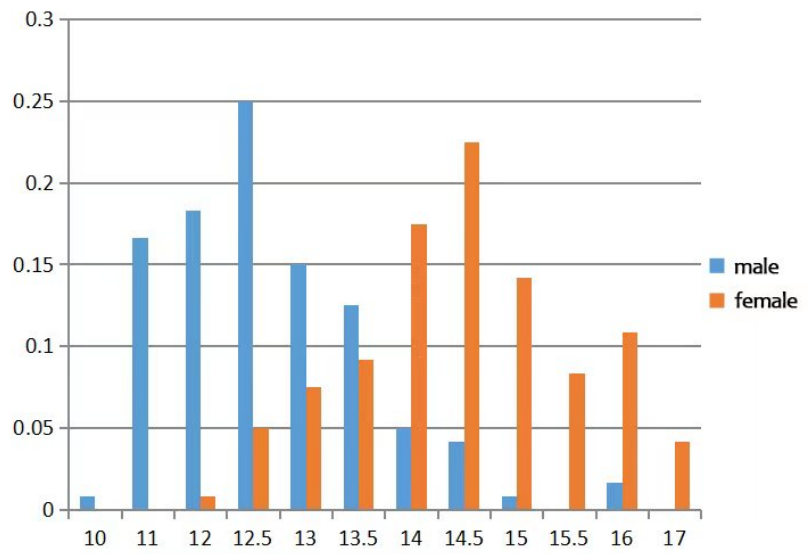

Figure 2 Mean frequency distribution of ten-fingerprint ridge between the males and the females

$\mathrm{x}$-axis: average of the ten-fingerprint ridge (ridges); $\mathrm{y}$-axis: percentage of the males and the females (\%) calculated. It was found that the 14.5 ridges $/ 25 \mathrm{~mm}^{2}$ or

\section{Results}

\subsection{Results of differences in fingerprint ridge thickness between the males and the females}

It has long been recognized that human fingers, palms and soles have ridged skin, which are characterized by a complex pattern of "mountains and valleys". These mountains are called the ridges and valleys are called furrows, and their ridge density is significantly higher than that of males $(\mathrm{p}<0.001)$. Two important factors determining ridge density are ridge thickness and furrow. Cummins and Ohler researched the ridge thickness of fingerprints and found that male ridges were thicker than those of the females, suggesting that males have fewer ridge than females in certain areas and therefore have less ridge density. [5] The results of this study were consistent with earlier reports that the ridge density of the females of different races was higher than that of the males. The experimental results showed that the difference between the average ridge density of females and males in the young Han population was 1.876 ridges $/ 25 \mathrm{~mm}^{2}$.

Ridge thickness and furrow are 2 important factors that determine the ridge density faults. Researches on fingerprint ridge thickness showed that males had thicker ridges than females, suggesting that males had fewer ridges than females in a particular area, and therefore had fewer ridges.

The results of this study and the frequency distribution of average ridge density are shown in Figure 2. It was observed that the average male ridge density of $88.3 \%$ of the Fujian Han young population was less than 14 ridges $/ 25 \mathrm{~mm}^{2}$, and greater than 14 ridges $/ 25 \mathrm{~mm}^{2}$ for $77.5 \%$ of females. It was observed that the average ridge density of males was not more than 16 ridges/ $25 \mathrm{~mm}^{2}$, and the average ridge density of females was not less than 12 ridges $/ 25 \mathrm{~mm}^{2}$.

The frequency distribution of probability density was used to calculate statistical analysis and dominance ratio, as shown in Figure 6, the subject's dominance ratio was less were more likely to be from the males, and the 12 density $/ 25 \mathrm{~mm}^{2}$ or more was more likely to be from the females.

\subsection{Correlation between the average density of ten-fingerprints and gender differences}

After statistical analysis of all the data, it was concluded that the ridge density of ten- fingerprint for the males was between $10-17$ ridges $/ 25 \mathrm{~mm}^{2}$, and the average ten-fingerprint density (XR) was 12.87 ridges $/ 25 \mathrm{~mm}^{2}$; and the ridge density of the females was between 12-17.5 ridges $/ 25 \mathrm{~mm}^{2}$, and the average ten-fingerprint density (XR) was 14.746 ridges $/ 25 \mathrm{~mm}^{2}$ (see Table 2 ). And after statistics, there is also a conclusion about the distribution of the average density of ten- fingerprints. Approximately $95 \%$ of the males' average tenfingerprint density was smaller than 14.5 ridges $/ 25 \mathrm{~mm}^{2}$, and approximately $77.5 \%$ of the females' ten- fingerprint density was greater than 14 ridges $/ 25 \mathrm{~mm}^{2}$ (see Table 1 , Table 3 ). If the average ridge density is smaller than 14.5 ridges $/ 25 \mathrm{~mm}^{2}$, then the possibility that this fingerprint comes from a man $(\mathrm{P}=0.95)$ is greater; if the average ridge density is greater than 14 ridges $/ 25 \mathrm{~mm}^{2}$, then the possibility that this fingerprint comes from a woman $(\mathrm{P}=0.775)$ is greater (see Table 1, Table 3, and Figure 2). After counting the average of the density of the ridge of the both five- fingerprint of the males and the females, it was obtained that the average density of five fingerprints of male hands (thumb, index finger, middle finger, ring finger, pinky finger) was always lower than 14 ridges $/ 25 \mathrm{~mm}^{2}$; the average density of five fingerprints of female hands (thumb, index finger, middle finger, ring finger, pinky finger) was always greater than 14 ridges $/ 25 \mathrm{~mm}^{2}$ (see Table 5). This was the difference between the males and the females after averaging large data. Therefore, it was considered that the average fingerprint density value of XR: The average density value of ten-fingerprints had a certain correlation with the gender difference. 


\section{Discussion}

Dermatology is the important research content in genetics, anthropology, and dactylography. Fingerprints are unique, they represent everyone's unique biological characteristics, and are called "exposed genetic factors." Diverse fingerprint types are an external dermatoglyphic form, and also the most regular and important manifestation. They are the human's unique trait, which are heritable between two generations, and are the only ones with uniqueness and relative stability. Among different groups and individuals, their performance traits are different, which is a unique genetic marker for humans. [6]

When fingerprint studies are used on the population, although relatively few studies have experienced assessing the gender difference in the thickness of the human epidermis ridges, they have proven to be a useful inference tool in gender differences in potential fingerprints from unknown sources. As Jantz and Owsley said, these differences reflected the fact that the radial and ulnar sides of the fingers were clearly responsive to different developmental instructions. This has proved that as a separate variable dermatoglyph analysis and support for topological classification systems, the use of radius and ulna is at least suitable for fingers. The distribution pattern of fingerprint ridge density is constant in all regions, and male and female fingers are very similar, although with different quantifications. It must be noted gender differences were found to be significant or not in the distal region (radial and ulnar regions) but not in the proximal region (lower region).

From the results, it can be concluded that there is a clear difference between male and female fingerprints. The females' ten-fingerprint ridge density is generally higher than that of the males. And this conclusion is generally consistent with the findings of Vinod C. But in details, it is different from the findings of (Vinod C): among the young Han population in Fujian, if XR is less than 12 ridges $/ 25 \mathrm{~mm}^{2}$, it is likely to be male, and if XR is more than 13 ridges $/ 25 \mathrm{~mm}^{2}$, it is likely to be female, which may be due to the limited samples and small errors from the human eyes when counting. At present, it is not possible to explain specifically why fingerprint density differs between males and females. But there is a point that the genes controlling fingerprint ridges may be related to the $\mathrm{x}$ chromosome, and this gene is dominant on the chromosome. Because female genes have two $\mathrm{x}$ chromosomes, the genes that control fingerprint ridges are double-expressed, so the density is greater than that of the males with only one $\mathrm{x}$ chromosome. However, relevant research experts also said that the difference in fingerprint density between the males and the females is the result of the combined effects of height, body shape, daily life, work environment, and regulation of estrogen; and genes are only part of the factor. However, no matter what causes the density difference of fingerprint ridges between males and females, this difference is real and credible.

Next, the research will focus on the large-scale investigations by using the experimental results. The sample sizes will be increased. The study of the differences in fingerprint patterns will be extended to among nations and among groups. In the near future, the difference in the density of fingerprint ridge between the males and the females may be widely used to identify suspects at the crime scene, and this research will also provide more clues to the investigation.

\section{References}

1. Weiping Guo. The detailed features of a fingerprint ridge-Research on the application of "discontinuity" in fingerprint examination[J]. Criminal technology, 2017, 42(03): 199-202.

2. M A Acree.Is there a gender difference in fingerprint ridge density[J].Forensic Sci.Int, 1999,102:35-44.

3. Pingya Ni, Wendong GE, Li Fei.A preliminary study on the gender difference of ridge density in fingerprints [J].Criminal technology, 2012(02): 3-5.

4. Vinod C Nayak, Prateek Rastogi, Tanuj Kanchan,et al.Sex differences from fingerprint ridge density in Chinese and Malaysian population [J]. Forensic Science International, 2010, 197:67-69.

5. V C Nayak,Prateek Rastogi, Tanuj Kanchan,et al. Sex differences from fingerprint ridge density in the Indian population[J]. Journal of Forensic and Legal Medicine,2010,17: 84-86.

6. Jianjun Gan, Xiankan Liu, Duan Yang, Kedi Zhu.Comparative analysis of fingerprint patterns between han and yi people in honghe prefecture, yunnan province[J].Journal of China criminal police college,2018(02): 142. 